\title{
QUASICONFORMAL EXTENSION OF MEROMORPHIC FUNCTIONS WITH NONZERO POLE
}

\author{
B. BHOWMIK *, G. SATPATI, AND T. SUGAWA
}

\begin{abstract}
In this note, we consider meromorphic univalent functions $f(z)$ in the unit disc with a simple pole at $z=p \in(0,1)$ which have a $k$-quasiconformal extension to the extended complex plane $\widehat{\mathbb{C}}$, where $0 \leq k<1$. We denote the class of such functions by $\Sigma_{k}(p)$. We first prove an area theorem for functions in this class. Next, we derive a sufficient condition for meromorphic functions in the unit disc with a simple pole at $z=p \in(0,1)$ to belong to the class $\Sigma_{k}(p)$. Finally, we give a convolution property for functions in the class $\Sigma_{k}(p)$.
\end{abstract}

\section{INTRODUCTION}

Let $\mathbb{C}$ denote the complex plane and $\widehat{\mathbb{C}}$ denote the extended complex plane $\mathbb{C} \cup$ $\{\infty\}$. We shall use the following notations: $\mathbb{D}=\{z:|z|<1\}, \overline{\mathbb{D}}=\{z:|z| \leq 1\}$, $\mathbb{D}^{*}=\{z:|z|>1\}, \overline{\mathbb{D}^{*}}=\{z:|z| \geq 1\}$. Let $f$ be a meromorphic and univalent function in the unit disk $\mathbb{D}$ with a simple pole at $z=p \in[0,1)$ of residue 1. Since $f(z)-1 /(z-p)$ is analytic in $|z|<1$, one has an expression of the form

$$
f(z)=\frac{1}{z-p}+\sum_{n=0}^{\infty} a_{n} z^{n}
$$

in $|z|<1$. We denote the class of such functions by $\Sigma(p)$. Let $\Sigma^{0}(p)$ be the subclass of $\Sigma(p)$ consisting of those functions $f$ for which $a_{0}=0$ in the above expansion. Note that if $f, g \in \Sigma^{0}(p)$ are related by $g=M \circ f$ for a Möbius transformation $M$, then $f=g$.

For a given number $0 \leq k<1, \Sigma_{k}(p)$ stands for the class of those functions in $\Sigma(p)$ which admit $k$-quasiconformal extension to the extended plane $\widehat{\mathbb{C}}$. Here, a mapping $F: \widehat{\mathbb{C}} \rightarrow \widehat{\mathbb{C}}$ is called $k$-quasiconformal if $F$ is a homeomorphism and has locally $L^{2}$-derivatives on $\mathbb{C} \backslash\left\{F^{-1}(\infty)\right\}$ (in the sense of distribution) satisfying $|\bar{\partial} F| \leq k|\partial F|$ a.e., where $\partial F=\partial F / \partial z$ and $\bar{\partial} F=\partial F / \partial \bar{z}$. Note that such an $F$ is called $K$-quasiconformal more often, where $K=(1+k) /(1-k) \geq 1$, in the literature. The quantity $\mu=\bar{\partial} F / \partial F$ is called the complex dilatation of $F$. See the

2010 Mathematics Subject Classification. 30C62, 30C55.

Key words and phrases. Quasiconformal map, Convolution

* Corresponding author.

The first author of this article would like to thank NBHM, DAE, India (Ref.No.2/48(20)/2012/NBHM(R.P.)/R\&D II/14916) for its financial support and the third author would like to thank JSPS Grant-in-Aid for Scientific Research (B) 22340025 for its partial financial support. 
standard textbook [5] by Lehto and Virtanen for basic properties of quasiconformal mappings. Set $\Sigma_{k}^{0}(p)=\Sigma^{0}(p) \cap \Sigma_{k}(p)$.

O. Lehto [3] refined the Bieberbach-Gronwall area theorem to the functions in $\Sigma_{k}(0)$ in the following form.

Theorem A. Let $0 \leq k<1$. Suppose that $f(z)=z^{-1}+a_{0}+a_{1} z+a_{2} z^{2}+\ldots$ is a function in $\Sigma_{k}(0)$. Then

$$
\sum_{n=1}^{\infty} n\left|a_{n}\right|^{2} \leq k^{2} .
$$

Here, equality holds if and only if

$$
f(z)=\frac{1}{z}+a_{0}+a_{1} z, \quad|z|<1,
$$

with $\left|a_{1}\right|=k$. Moreover, its $k$-quasiconformal extension is given by setting

$$
f(z)=\frac{1}{z}+a_{0}+\frac{a_{1}}{\bar{z}} \quad \text { for }|z| \geq 1 .
$$

On the other hand, the area theorem was extended by P. N. Chichra [1] to functions in $\Sigma(p)$ as follows.

Theorem B. Let $f \in \Sigma(p)$ have the expansion in (1.1). Then

$$
\sum_{n=1}^{\infty} n\left|a_{n}\right|^{2} \leq \frac{1}{\left(1-p^{2}\right)^{2}} .
$$

Equality holds for the function

$$
f_{p}(z)=\frac{1}{z-p}+a_{0}+\frac{z}{1-p^{2}}
$$

Our first result establishes an area theorem for the class $\Sigma_{k}(p)$. Interestingly, the form of extremal functions is different from that of the function $f_{p}$ in Theorem $\mathrm{B}$.

Theorem 1. Let $0 \leq k<1$ and $0 \leq p<1$. Suppose that $f \in \Sigma_{k}(p)$ is expressed in the form of (1.1). Then

$$
\sum_{n=1}^{\infty} n\left|a_{n}\right|^{2} \leq \frac{k^{2}}{\left(1-p^{2}\right)^{2}} .
$$

Here, equality holds if and only if $f$ is of the form

$$
f(z)=\frac{1}{z-p}+a_{0}+\frac{a_{1} z}{1-p z} \quad \text { for }|z|<1,
$$

where $a_{0}$ and $a_{1}$ are constants with $\left|a_{1}\right|=k$. Moreover, a $k$-quasiconformal extension of this $f$ is given by setting

$$
f(z)=\frac{1}{z-p}+a_{0}+\frac{a_{1}}{\bar{z}-p} \quad \text { for }|z| \geq 1 .
$$


Observe that this is a natural extension of Theorem A. We remark that the function in (1.4) belongs to $\Sigma(p)$ as long as $\left|a_{1}\right| \leq 1$ (see the latter part of the proof of Theorem 11 below). This function with $\left|a_{1}\right|=1$ provides another extremal case in (1.2). As an immediate corollary of the theorem, we obtain the following.

Corollary 1. Let $0<p<1$ and $0<k<1$. For $f \in \Sigma_{k}(p)$ with the expansion (1.1), the following inequality holds:

$$
\left|a_{1}\right|<\frac{k}{1-p^{2}}
$$

Note that the inequality $\left|a_{1}\right| \leq 1 /\left(1-p^{2}\right)$ for $f \in \Sigma(p)$ is sharp in view of Theorem B. We have no exact value of the best upper bound, say, $M(p, k)$ of $\left|a_{1}\right|$ for $f \in \Sigma_{k}(p)$. The extremal function in Theorem 1 and compactness of the class $\Sigma_{k}^{0}(p)$ yield, at least, the estimates $k \leq M(p, k)<k /\left(1-p^{2}\right)$ for $p, k \in(0,1)$.

Secondly, we provide a sufficient condition for functions of the form (1.1) to belong to the class $\Sigma_{k}(p)$.

Theorem 2. Let $0 \leq k<1$ and $0 \leq p<1$. Suppose that $\omega$ is an analytic function in $\mathbb{D}$ such that $\left|\omega^{\prime}(z)\right| \leq k(1+p)^{-2}$ for $z \in \mathbb{D}$. Then the function $f$ given by

$$
f(z)=\frac{1}{z-p}+\omega(z), \quad|z|<1
$$

is a member of $\Sigma_{k}(p)$. A k-quasiconformal extension is given by setting

$$
f(z)=\frac{1}{z-p}+\omega(1 / \bar{z}), \quad \text { for }|z|>1 .
$$

We note that J. G. Krzyż [2] proved this theorem when $p=0$. He also gave a convolution theorem in the same paper [2]. We can also extend it to a modified convolution. The modified Hadamard product (or the modified convolution) $f \star g$ of two functions $f, g \in \Sigma(p)$ with expansions

$$
f(z)=\frac{1}{z-p}+\sum_{n=0}^{\infty} a_{n} z^{n} \quad \text { and } \quad g(z)=\frac{1}{z-p}+\sum_{n=0}^{\infty} b_{n} z^{n}, \quad z \in \mathbb{D}
$$

is defined by

$$
(f \star g)(z)=\frac{1}{z-p}+\sum_{n=0}^{\infty} a_{n} b_{n} z^{n}, \quad z \in \mathbb{D} .
$$

Our third result concerns this Hadamard product.

Theorem 3. Let $f \in \Sigma_{k_{1}}(p)$ and $g \in \Sigma_{k_{2}}(p)$ for some $k_{1}, k_{2}, p \in[0,1)$. If $\alpha=$ $k_{1} k_{2}(1-p)^{-2}<1$, then the modified Hadamard product $f \star g$ belongs to $\Sigma_{\alpha}(p)$.

As we mentioned above, this result reduces to a theorem due to Krzyż [2] when $p=0$.

We prove Theorem 1 in Section 2 and Theorems 2 and 3 in Section 3. We also give another proof of a part of Theorem 1 as a concluding remark in Section 3. 


\section{Proof of Theorem 1}

We start the section with proving the area theorem for functions in the class $\Sigma_{k}(p)$. We follow the idea due to Lehto [3].

Proof of Theorem 1. Let $f \in \Sigma_{k}(p)$ have the expansion in (1.1). We may suppose that $f$ is already extended to a $k$-quasiconformal mapping of $\widehat{\mathbb{C}}$ onto itself. If $k=0$, then the assertion clearly holds. Hence, we assume that $k>0$ in the rest of the proof. To start with, we first make a change of variables. We define $\phi: \widehat{\mathbb{C}} \rightarrow \widehat{\mathbb{C}}$ by $\phi(\zeta)=f(1 / \zeta)$. Note that $\phi$ has locally $L^{2}$-derivatives on $\mathbb{C} \backslash\left\{\phi^{-1}(\infty)\right\}=\mathbb{C} \backslash\{1 / p\}$. Since the function $\psi(\zeta)=\phi(\zeta)-\zeta /(1-p \zeta)$ has the expression

$$
\psi(\zeta)=\phi(\zeta)-\frac{\zeta}{1-p \zeta}=\sum_{n=0}^{\infty} \frac{a_{n}}{\zeta^{n}}, \quad|\zeta|>1,
$$

and, in particular, is bounded and analytic near the point $\zeta=1 / p$, the function $\psi$ has locally $L^{2}$-derivatives on $\mathbb{C}$. Therefore for every $r>0$, we can apply the CauchyPompeiu formula (see [5, III §7] for details) to the function $\psi$ in the disk $|\zeta|<r$ to obtain

$$
\psi(\zeta)=\frac{1}{2 \pi i} \int_{|w|=r} \frac{\psi(w)}{w-\zeta} d w-\frac{1}{\pi} \iint_{|w|<r} \frac{\bar{\partial} \psi(w)}{w-\zeta} d u d v
$$

where $w=u+i v$. We note that $\psi(\zeta) \rightarrow a_{0}$ as $\zeta \rightarrow \infty$ and $\bar{\partial} \psi(\zeta)=0$ for $|\zeta|>1$. Letting $r \rightarrow+\infty$, we thus get

$$
\psi(\zeta)=a_{0}-\frac{1}{\pi} \iint_{|w|<1} \frac{\bar{\partial} \psi(w)}{w-\zeta} d u d v, \quad \zeta \in \mathbb{C} .
$$

We differentiate the above expression with respect to $\zeta$ and obtain

$$
\partial \psi(\zeta)=-\frac{1}{\pi} \iint_{|w|<1} \frac{\bar{\partial} \psi(w)}{(w-\zeta)^{2}} d u d v=H[\bar{\partial} \psi](\zeta), \quad \zeta \in \mathbb{C},
$$

where $H$ is the two dimensional Hilbert transformation. (Strictly speaking, the above integral should be understood as Cauchy's principal value for $|\zeta| \leq 1$. See [5, III $\S 7]$ for details.) Since $H$ is a linear isometry of $L^{2}(\mathbb{C})$, in conjunction with (2.3), we have

$$
\iint_{\mathbb{D}}|\bar{\partial} \psi(\zeta)|^{2} d \xi d \eta=\iint_{\mathbb{C}}|H[\bar{\partial} \psi](\zeta)|^{2} d \xi d \eta=\iint_{\mathbb{C}}|\partial \psi(\zeta)|^{2} d \xi d \eta,
$$

where $\zeta=\xi+i \eta$. Next, we recall that Chichra indeed showed the following relation in the proof of Theorem B:

$$
\operatorname{Area}(\mathbb{C} \backslash f(\mathbb{D}))=\pi\left[\frac{1}{\left(1-p^{2}\right)^{2}}-\sum_{n=1}^{\infty} n\left|a_{n}\right|^{2}\right] .
$$


We remark that $f(\partial \mathbb{D})$ is of area zero because $f$ is quasiconformal. Noting $\phi(\mathbb{D})=$ $f\left(\mathbb{D}^{*}\right)=\mathbb{C} \backslash f(\overline{\mathbb{D}})$, we thus have the relation

$$
\text { Area } \phi(\mathbb{D})=\pi\left[\frac{1}{\left(1-p^{2}\right)^{2}}-\sum_{n=1}^{\infty} n\left|a_{n}\right|^{2}\right] \text {. }
$$

Since $|\bar{\partial} \phi| \leq k|\partial \phi|$ a.e., the Jacobian $J_{\phi}$ of $\phi$ satisfies the inequality

$$
J_{\phi}=|\partial \phi|^{2}-|\bar{\partial} \phi|^{2} \geq\left(1-k^{2}\right)|\partial \phi|^{2} \geq\left(k^{-2}-1\right)|\bar{\partial} \phi|^{2}=\left(k^{-2}-1\right)|\bar{\partial} \psi|^{2} .
$$

Hence, we obtain

$$
\operatorname{Area} \phi(\mathbb{D})=\iint_{\mathbb{D}} J_{\phi}(\zeta) d \xi d \eta \geq\left(k^{-2}-1\right) \iint_{\mathbb{D}}|\bar{\partial} \psi(\zeta)|^{2} d \xi d \eta
$$

Next, we see from (2.4) that

$$
\iint_{\mathbb{D}}|\bar{\partial} \psi(\zeta)|^{2} d \xi d \eta=\iint_{\mathbb{C}}|\partial \psi(\zeta)|^{2} d \xi d \eta \geq \iint_{|\zeta|>1}|\partial \psi(\zeta)|^{2} d \xi d \eta .
$$

It is easy to evaluate the right-most integral above by using the expansion in (2.1) as follows:

$$
\iint_{|\zeta|>1}|\psi(\zeta)|^{2} d \xi d \eta=\pi \sum_{n=1}^{\infty} n\left|a_{n}\right|^{2}
$$

Plugging this with (2.5), (2.6) and (2.7), we obtain

$$
\left(k^{-2}-1\right) \pi \sum_{n=1}^{\infty} n\left|a_{n}\right|^{2} \leq \pi\left[\frac{1}{\left(1-p^{2}\right)^{2}}-\sum_{n=1}^{\infty} n\left|a_{n}\right|^{2}\right],
$$

which yields the desired inequality.

Finally, we analyze the equality case for (1.3). Suppose that equality holds in (1.3). Then, equalities must hold both in (2.6) and in (2.7). The equality in (2.7) implies that $\partial \psi=0$ on $\mathbb{D}$. In other words, $h=\bar{\psi}$ is analytic on $\mathbb{D}$. Therefore, $\phi(\zeta)=\zeta /(1-p \zeta)+\overline{h(\zeta)}$. The equality in (2.6) means that $|\bar{\partial} \phi / \partial \phi|$ is the constant $k$ a.e. on $\mathbb{D}$. Since $\bar{\partial} \phi(\zeta) / \partial \phi(\zeta)=\overline{h^{\prime}(\zeta)}(1-p \zeta)^{2}$, it implies that the analytic function $h^{\prime}(\zeta)(1-p \zeta)^{2}$ has constant modulus $k$ and therefore a constant $\alpha$ with $|\alpha|=k$. Hence, $h^{\prime}(\zeta)=\alpha(1-p \zeta)^{-2}$ for $|\zeta|<1$. Integrating it, we obtain $h(\zeta)=\alpha \zeta /(1-p \zeta)+h(0)$. Thus, we finally have the form

$$
\phi(\zeta)=\frac{\zeta}{1-p \zeta}+\overline{h(0)}+\frac{\bar{\alpha} \bar{\zeta}}{1-p \bar{\zeta}}, \quad \zeta \in \mathbb{D}
$$

Therefore,

$$
f(z)=\frac{1}{z-p}+\overline{h(0)}+\frac{\bar{\alpha}}{\bar{z}-p}, \quad z \in \mathbb{D}^{*},
$$

whose boundary values on $\partial \mathbb{D}$ are the same as those of the meromorphic function

$$
g(z)=\frac{1}{z-p}+\overline{h(0)}+\frac{\bar{\alpha} z}{1-p z} .
$$


Since $f(z)-g(z)$ is bounded analytic on $\mathbb{D}, f(z)$ is identically equal to $g(z)$ on $\mathbb{D}$ by the maximum principle. In particular, $h(0)=\overline{a_{0}}$ and $\alpha=\overline{a_{1}}$. Thus we have seen that the function $f$ must have the form (1.4) if equality holds in (2.7). We need to show that the function $f$ of the form (1.4) is indeed a member of $\Sigma_{k}(p)$. We first show that $f$ is univalent in $\mathbb{D}$. We compute

$$
f\left(z_{1}\right)-f\left(z_{2}\right)=\frac{z_{2}-z_{1}}{\left(z_{1}-p\right)\left(z_{2}-p\right)}\left[1-a_{1}\left(\frac{z_{1}-p}{1-p z_{1}} \cdot \frac{z_{2}-p}{1-p z_{2}}\right)\right]
$$

for $z_{1}, z_{2} \in \mathbb{D}$. Since

$$
\left|a_{1}\left(\frac{z_{1}-p}{1-p z_{1}} \cdot \frac{z_{2}-p}{1-p z_{2}}\right)\right|<\left|a_{1}\right|=k \leq 1
$$

for $z_{1}, z_{2} \in \mathbb{D}$, we see that $f\left(z_{1}\right) \neq f\left(z_{2}\right)$ if $z_{1} \neq z_{2}$. Hence, $f \in \Sigma(p)$. On the other hand, the function in (1.5) agrees with that in (1.4) on the boundary $|z|=1$, and is a composition of the Möbius transformation $1 /(z-p)$ with the $k$-quasiconformal affine mapping $w+a_{0}+a_{1} \bar{w}$. Thus we conclude that $f$ belongs to $\Sigma_{k}(p)$.

\section{Proof of Theorems 2 and 3 And a CONCluding REMARK}

We start with the proof of Theorem 2 .

Proof of Theorem 2. We first investigate the behavior of the function $\omega$. Since $\left|\omega^{\prime}\right| \leq k(1+p)^{-2}$, we have the inequality

$$
\left|\omega\left(z_{1}\right)-\omega\left(z_{2}\right)\right| \leq \frac{k}{(1+p)^{2}}\left|z_{1}-z_{2}\right|, \quad z_{1}, z_{2} \in \mathbb{D} .
$$

We thus conclude that $\omega$ is (uniformly) Lipschitz continuous on $\mathbb{D}$. In particular, $\omega$ extends to $\overline{\mathbb{D}}$ continuously with the same Lipschitz constant. Also, the function $f(z)=1 /(z-p)+\omega(z)$ extends to $|z| \leq 1$ continuously. We now see that

$$
\left|\frac{1}{z_{1}-p}-\frac{1}{z_{2}-p}\right|=\frac{\left|z_{1}-z_{2}\right|}{\left|z_{1}-p\right|\left|z_{2}-p\right|} \geq \frac{\left|z_{1}-z_{2}\right|}{(1+p)^{2}}, \quad z_{1}, z_{2} \in \mathbb{D} .
$$

Hence, we have

$$
\left|f\left(z_{1}\right)-f\left(z_{2}\right)\right| \geq \frac{1-k}{(1+p)^{2}}\left|z_{1}-z_{2}\right|
$$

for $z_{1}, z_{2} \in \overline{\mathbb{D}}$, which proves that $f(z)$ is univalent on $|z| \leq 1$.

For a while, we denote by $g(z)$ the function appearing in the right-hand side of (1.6). Obviously, $g(z)$ can be defined in $|z| \geq 1$ and agrees with the above $f(z)$ on $|z|=1$. We show now that $g$ is $k$-quasiconformal on $|z|>1$. A computation yields

$$
\partial g(z)=\frac{-1}{(z-p)^{2}} \quad \text { and } \quad \bar{\partial} g(z)=\frac{-\omega^{\prime}(1 / \bar{z})}{\bar{z}^{2}} .
$$

Hence, the complex dilatation $\mu=\bar{\partial} g / \partial g$ of $g$ satisfies

$$
|\mu(z)|=\frac{|z-p|^{2}}{|z|^{2}}\left|\omega^{\prime}(1 / \bar{z})\right| \leq(1+p)^{2}\left|\omega^{\prime}(1 / \bar{z})\right| \leq k<1
$$


for $|z|>1$. Since the unit circle is removable for quasiconformality (see [5, p.205]), we conclude that $g$ gives a $k$-quasiconformal extension of $f$.

A straightforward application of Theorem 2 yields the following sufficient condition for a function $f$ of the form (1.1) to belong to $\Sigma_{k}(p)$.

Corollary 2. Let $0 \leq p<1$ and $0 \leq k<1$. Suppose that a meromorphic function $f(z)$ on $|z|<1$ has the form (1.1). If

$$
\sum_{n=1}^{\infty} n\left|a_{n}\right| \leq \frac{k}{(1+p)^{2}}
$$

then $f \in \Sigma_{k}(p)$.

Proof. This immediately follows from Theorem 2 because

$$
\left|\omega^{\prime}(z)\right| \leq \sum_{n=1}^{\infty} n\left|a_{n}\right||z|^{n-1} \leq \sum_{n=1}^{\infty} n\left|a_{n}\right| \leq \frac{k}{(1+p)^{2}}, \quad|z|<1 .
$$

Next we prove Theorem 3 .

Proof of Theorem 3. Let $f \in \Sigma_{k_{1}}(p)$ and $g \in \Sigma_{k_{2}}(p)$ be expressed as in (1.4). Then Theorem 1 gives us

$$
\sum_{n=1}^{\infty} n\left|a_{n}\right|^{2} \leq \frac{k_{1}{ }^{2}}{\left(1-p^{2}\right)^{2}} \quad \text { and } \quad \sum_{n=1}^{\infty} n\left|b_{n}\right|^{2} \leq \frac{k_{2}{ }^{2}}{\left(1-p^{2}\right)^{2}} .
$$

Now an application of Cauchy-Schwarz inequality together with the aforementioned inequalities yields

$$
\begin{aligned}
\sum_{n=1}^{\infty} n\left|a_{n} b_{n}\right| & =\sum_{n=1}^{\infty}\left(\sqrt{n}\left|a_{n}\right|\right)\left(\sqrt{n}\left|b_{n}\right|\right) \\
& \leq\left(\sum_{n=1}^{\infty} n\left|a_{n}\right|^{2}\right)^{1 / 2}\left(\sum_{n=1}^{\infty} n\left|b_{n}\right|^{2}\right)^{1 / 2} \\
& \leq \frac{k_{1} k_{2}}{\left(1-p^{2}\right)^{2}}=\frac{\alpha}{(1+p)^{2}}
\end{aligned}
$$

where $\alpha=k_{1} k_{2}(1-p)^{-2}$. Since $\alpha<1$ by assumption, the desired result follows from Corollary 2 ,

We conclude the present note with an outline of another proof of (1.3) based on Lehto's principle (cf. [4, II.3.3]) and Theorem B. Before it, we recall the definition of the complex Banach (indeed, Hilbert) space $\ell^{2}$. This is the set of sequences $x=$ $\left\{x_{n}\right\}_{n=1}^{\infty}$ of complex numbers with the norm

$$
\|x\|_{\ell^{2}}=\left(\sum_{n=1}^{\infty}\left|x_{n}\right|^{2}\right)^{1 / 2}<\infty
$$


It is enough to show (1.3) for functions in $\Sigma_{k}^{0}(p)$ only. Suppose that $f \in \Sigma_{k}^{0}(p)$ is already extended to a $k$-quasiconformal mapping of $\widehat{\mathbb{C}}$ and let $\mu$ be its complex dilatation. We remark that $|\mu| \leq k$ a.e. in $\mathbb{D}^{*}$ and $\mu=0$ in $\mathbb{D}$. By the measurable Riemann mapping theorem, for each $t \in \mathbb{D}$, there exists a unique quasiconformal mapping $f_{t}$ of $\widehat{\mathbb{C}}$ for which the complex dilatation is $t \mu / k$ and $\left.f_{t}\right|_{\mathbb{D}} \in \Sigma^{0}(p)$. Note here that $f_{k}=f$. Then $f_{t}$ has an expansion of the form

$$
f_{t}(z)=\frac{1}{z-p}+\sum_{n=1}^{\infty} a_{n}(t) z^{n}
$$

in $|z|<1$. By the holomorphic dependence of the solution to the Beltrami equation, $a_{n}(t)$ is analytic in $|t|<1$ for every $n \geq 1$. We now consider the sequence $\sigma(t)=$ $\left\{\sqrt{n} a_{n}(t)\right\}_{n=1}^{\infty}$. Theorem B tells us that $\|\sigma(t)\|_{\ell^{2}} \leq 1 /\left(1-p^{2}\right)$. Hence, we conclude that $\sigma: \mathbb{D} \rightarrow \ell^{2}$ is a bounded analytic function taking values in the complex Banach space $\ell^{2}$. Since $\sigma(0)=0$, the (generalized) Schwarz lemma yields the inequality $\|\sigma(t)\|_{\ell^{2}} \leq|t| /\left(1-p^{2}\right)$. In particular, letting $t=k$ gives (1.3) .

We must say that this method is conceptually simpler than that of our proof in Section 2. However, this does not provide information about the equality case in an obvious manner.

Acknowledgement: The authors thank Karl-Joachim Wirths for his suggestions and careful reading of the manuscript.

\section{REFERENCES}

1. P. N. Chichra, An area theorem for bounded univalent functions, Proc. Cambridge Philos. Soc. 66 (1969), 317-321.

2. J. G. Krzyż, Convolution and quasiconformal extension, Comment. Math. Helv. 51 (1976), 99-104.

3. O. Lehto, Schlicht functions with a quasiconformal extension, Ann. Acad. Sci. Fenn. Ser. A I (1971), no. 500, 10.

4. _ Univalent Functions and Teichmüller Spaces, Springer-Verlag, 1987.

5. O. Lehto and K. I. Virtanen, Quasiconformal Mappings in the Plane, 2nd Ed., Springer-Verlag, 1973 .

Bappaditya Bhowmik, Department of Mathematics, Indian Institute of TechnolOGY KHARAGPUR, KharagPUR - 721302, INDIA.

E-mail address: bappaditya@maths.iitkgp.ernet.in

Goutam Satpati, Department of Mathematics, Indian Institute of Technology KHARAGPUR, KharagPuR - 721302, India.

E-mail address: goutam.satpati@gmail.com

Toshiyuki Sugawa, Graduate School of Information Sciences, Tohoku University, AOBA-KU, SEndai 980-8579, JAPAN

E-mail address: sugawa@math.is.tohoku.ac.jp 